\title{
Evaluation Framework for Passive Assistive Device Based on Humanoid Experiments
}

\author{
Yumeko Imamura*, Ko Ayusawa ${ }^{\dagger}$ and Eiichi Yoshida* \\ CNRS-AIST Joint Robotics Laboratory (JRL), UMI3218/RL, \\ Intelligent Systems Research Institute, \\ National Institute of Advanced Industrial Science and Technology (IS-AIST), \\ 1-1-1, Umezono, Tsukuba 305-8560, Japan \\ Takayuki Tanaka \\ Graduate School of Information Science and Technology, \\ Hokkaido University, N14-W9, Kita-ku, Sapporo 060-0814, Japan \\ ttanaka@ssi.ist.hokudai.ac.jp
}

Received 10 April 2017

Accepted 1 August 2017

Published 5 October 2017

\begin{abstract}
This study presents an enhanced framework for evaluating an assistive effect generated by a passive assistive device using a humanoid robot. The humanoid robotic experiments can evaluate wearable devices by measuring the joint torque, which cannot be measured directly from the human body. In this paper, we introduce an "assistive torque estimation map" as an efficient means for estimating the supportive torque within the range of motions by interpolating the measured joint torques and joint angles of the robot. This map aims to estimate the supportive torques for complex motions without conducting humanoid experiments or human-subject experiments with these motions. We generated an estimation map for an actual assistive suit that decreases the load on the lumbar region and we verified the validity of the proposed method by experimentation. In addition, the geometric simulation model of the assistive suit was validated based on the proposed experiments by using the humanoid robot HRP-4. The proposed framework is expected to lead to an efficient design of such assistive devices so that fewer human-subject experiments need to be conducted.
\end{abstract}

Keywords: Humanoid robot; assistive device evaluation.

${ }^{*}$ Corresponding author.

This is an Open Access article published by World Scientific Publishing Company. It is distributed under the terms of the Creative Commons Attribution 4.0 (CC-BY) License. Further distribution of this work is permitted, provided the original work is properly cited. 


\section{Y. Imamura et al.}

\section{Introduction}

In rapidly aging societies, such as the Japanese society, various types of assistive devices are being invented to support human movements and worker's tasks. In recent years, wearable assistive devices have been attracting attention and some such devices have already been produced commercially. Sankai developed an exoskeletal robot suit called hybrid assistive limb (HAL). ${ }^{1}$ HAL was developed to extend and amplify human physical functions by using multiple sensors and motors. The effect of HAL in reducing the level of muscle activity has been verified and it is currently being used in rehabilitation programs. Kobayashi developed a "muscle suit" 2 that enables manual workers to lift and carry heavy weights by supporting the back muscles using the McKibben artificial muscles. Besides this, there are many kinds of exoskeletal and orthotic devices, ${ }^{3}$ such as the load-carrying exoskeleton Berkeley lower extremity exoskeleton (BLEEX), ${ }^{4}$ the exoskeletons for upper limb, ${ }^{5}$ and the semi-active assistive system "Smart Suit" ${ }^{6}$

These assistive devices have a high degree of contact with humans; therefore, there is a need for careful verification before actually introducing them commercially. The international safety standard ISO 13482, published in 2014, was the first evaluation criteria regarding safety features, such as electromagnetic interference, durability, and functional safety. ${ }^{7}$ Following it, we need to establish now a standardized scheme for the quantitative evaluation of the assistive performance for humans.

There has been continuous progress in the development of wearable-type devices; however, evaluating these devices quantitatively has been a challenge. One solution is to simulate the human and the device by constructing digital models. The design and control of the assistive systems are often performed by modeling humans as multibody systems. ${ }^{8}$ In this method, although there is a validity problem in modeling humans, it is necessary to validate the modeling of the devices.

The other evaluation method is the human-subject experiment. The most common approach for this is surface electromyography (EMG) measurement ${ }^{9}$ because it is a non-invasive measurement, and many devices aim at reducing the muscle burden. There are also other non-invasive evaluation reports such as the assessment of muscle fatigue by near-infrared spectroscopy, ${ }^{10}$ the enhancement in force perception, ${ }^{11}$ the improvement in trunk stability, ${ }^{12}$ and the subjective evaluation by users. However, human measurements have several bottlenecks, such as individual differences, the necessity of making many assumptions about mechanics and physiology, reproducibility, and ethical concerns. Furthermore, it is difficult to measure the internal forces such as the joint torques or muscle tensions. In general, they are estimated by measuring the motion and the contact forces and by analyzing these data with the models. The estimation involving multiple contact points also suffers from the redundancy problem, besides the validity problem seen in the digital human model. The experiment also requires a large number of trials because of low reproducibility. Therefore, it is difficult to obtain quantitative data sufficient for the verification of the devices through human-subject experiments.

In previous studies, we have proposed the verification of the devices by using humanoid robots. ${ }^{13-16}$ Humanoid robots fitted with internal sensors provide the 
values of internal forces, which are difficult to measure in human-subject experiments. Humanoid robots have body shapes similar to humans, and they are able to wear assistive devices and interact with their assistive forces. In addition, humanoid robots have advantages such as their ability to imitate human motions and to repeat the same motions precisely, which provides reproducibility; they can also be used as simulators that imitate humans in the real environment. In a related research, Kondo et al. developed a humanoid bipedal robot WABIAN-2R as a human motion simulator. ${ }^{17}$ They performed humanoid walking experiments using a walking-aid device in which the users grip the handle to support their bodies. The results showed the relationship between the walking posture and the energy consumption at the knee joints, which is consistent with the clinical knowledge. Boston Dynamics developed an anthropometric robot PETMAN to test the chemical protective clothing. ${ }^{18}$ PETMAN can walk and do basic calisthenics on a treadmill in the wind tunnel chamber. The robot can control the skin temperature and the sweating rate, and the chemical sensor will detect chemical agents if they are within the clothing.

In our previous report, ${ }^{15}$ the effect of a power-assistive supporter Smart Suit Lite $(\mathrm{SSL})^{19}$ was evaluated with the humanoid robot, and we confirmed that a moment arm estimated from the measured assistive forces and the joint torque was consistent with a robot geometric model. The other paper $^{16}$ reported that we estimated supporting torques by using identification techniques of the robot's mechanical properties, and showed that the mechanical properties of the assistive device can be identified with the humanoid experiments when assuming the assistive device as a certain wire-driven multi-body systems. These papers showed the validity of the evaluation of the assistive effects with the humanoid robot.

These previous works evaluated a few specific movements mimicking human motions. When we want to know the effect for another motion which has not been measured yet, the motion generation and the measurement experiment by using the humanoid robot are required each time.

In this paper, based on those results, we propose a more general method to quantitatively evaluate the assist characteristics of the assistive devices. The differences with the previous works is that the proposed method does not utilize a motion which mimicks a specific human motion and does not use prior knowledge or a model of an assist mechanism. This approach constructs the estimation model, which is named the assistive torque estimation map, from the measurement data of the basic postures of the humanoid robot. This paper also shows the results of estimating the assistive effects during complex motions with this estimation map.

The method targets the "passive assistive device" whose supporting power during motions is determined only by the posture of the user and not influenced by external forces and biological signals. The passive assistive device commonly obtains assistive forces with passive elements such as mechanical springs, elastic fibers, and air springs. This paper assumed the movements using the chest joint mainly, and applied the method to generate an estimation model of the assistive effect on the chest joint. 


\section{Y. Imamura et al.}

\section{Evaluation of an Assistive Device Using a Humanoid Robot}

\subsection{Humanoid robot as a device evaluator}

Although the most basic method when evaluating the device is the human-subject experiment, it has several drawbacks, such as individual differences in physique, repeatability of motions, and ethical procedures. The technique of numerical simulation is often employed for these reasons. The simulation allows the designers to change the physical properties of the human body and the design parameters of the device. Such a model-based approach is widely used. The most critical problem here is the validity of the mechanical effects, such as the contact force, in case of close interactions between the human being and the device.

To overcome those issues, we employed a humanoid robot instead of human subjects to evaluate the assistive device and its simulation model quantitatively. The advantages of using a humanoid robot are as follows ${ }^{13}$ :

- Humanoid robots have a morphology similar to humans; therefore, they can physically simulate the use of the device in real life in a manner similar to humans.

- Humanoid robots can repeat exactly the same motions and provide quantitative measures, such as joint trajectories, torques, and applied forces.

- Experiments using humanoid robots are free of ethical concerns arising from the risk of injury.

Figure 1 shows how the humanoid robot can be integrated in the framework of the product design and evaluation of the assistive devices.

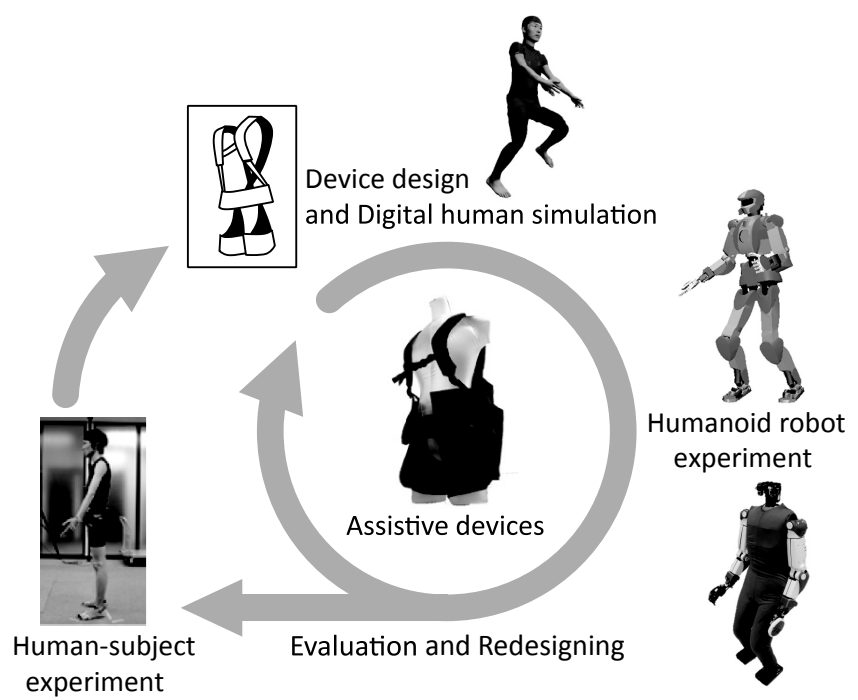

Fig. 1. Design and evaluation framework. Experiment with humanoid robot aims to provide data for quantitative evaluation and redesign with fewer human-subject experiments. 
The humanoid robot used for this objective has several requirements. First, the robot needs to have a shape similar to humans. The body shape and the mass of the robot should be similar to those of the user to wear devices designed for humans and to observe the expected effect. In this research, we used a humanoid robot HRP$4^{21}$ wearing a soft suit instead of a hard plastic cover to realize a soft surface similar to a human being (Fig. 2). HRP-4 is $1.51 \mathrm{~m}$ in height and $39 \mathrm{~kg}$ in weight; the geometric parameters of HRP-4 are close to those of an average young Japanese woman. ${ }^{22}$

Second, the robot is required to have kinematics close to humans. The skeleton and joints of the robot are simplified and different from the precise configuration of the human body. However, in the field of biomechanical analysis, a simplified skeleton model is widely used. ${ }^{23}$ In particular, for model-based designs, such simplified models are often used because of some practical issues. ${ }^{24} \mathrm{HRP}-4$ can be used as a physical simulator corresponding to such biomechanical models.

\subsection{Assistive torque estimation map}

This section introduces a method for obtaining the relationship between the joint angles and the assistive effects of a passive assistive device by measuring the joint torques of the humanoid robot. The proposed method is performed as follows:

(i) Creation of a set of postures

A set of postures for joint torque measurement is created to cover the range of motions of the target joint or at least to cover the joint angles used in the target motions. Several angles are determined discretely in the range of motion and threedimensional movements are generated as a combination of these angles.

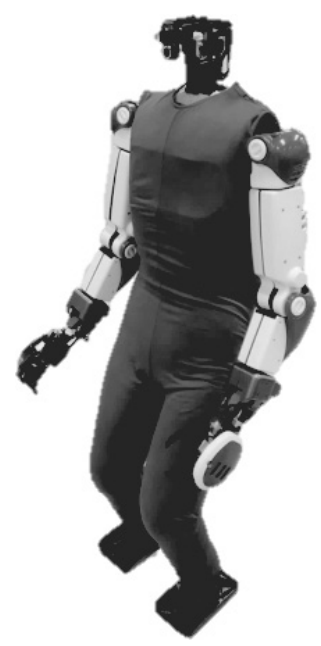

Fig. 2. HRP-4 with a soft body surface. 
(ii) Measurement of the joint torque

The joint torques during the movements created in the preceding paragraph are measured using the humanoid robot. The measurements are performed with the same movement for each condition, with or without assistance.

(iii) Creation of a relational expression

A relational expression between the torques and the angles is assumed and their parameters are determined based on the torque measurement data for each assist condition. These estimation equations are defined as the torque estimation maps on the target joint. The difference between the estimates under the condition with and without assistance is the assistive effect. In the present study, we referred to the difference as the assistive torque estimation map.

This approach can construct the estimation model directly from the measurement data without using any specific motions, human-subject experiments, or simulation models of the device. Based on the estimation map, the assistive force generated at any posture can be estimated. However, there is a possibility of an estimation error occurring when the target motion is fast, because the map is created using quasi-static measurements. In addition, if too many degrees of freedom need to be focused on, the number of the combinations of the joint angles becomes very high. Then, measuring and modeling all conditions becomes difficult. Therefore, considering whether constructing an estimation map with fewer combinations or extrapolations is reasonable is required.

\section{Assistive Torque Map Based on Actual Measurements}

We created an assistive torque estimation map of the SSL. Section 3.1 describes the target assistive suit, and Sec. 3.2 describes the experiment and construction of the estimation map. Furthermore, we compared the value estimated by using the created map and the measured value of the complex motion; this confirmed the usefulness of the proposed method.

\subsection{Power-assist device SSL}

This section explains the SSL and its design principle proposed in the previous work. ${ }^{19}$ Backaches are a common disorder in workers who are routinely involved in manual labor, such as care workers, agricultural workers, and industrial workers. The SSL aims at the reduction in the back muscle burden and the prevention of lumbar disorders by using the tension generated by elastic materials (as shown in Fig. 3). The elastic material on the shoulder is fixed to the vest and is connected to the elastic material on the opposite leg by the non-elastic belt. The length of the non-elastic belt is adjustable. These elastic belts generate assistive torques along with the change in the posture angle of the wearer. The assistive force generated by this structure helps the wearer to return to the upright position, and it reduces the burden on the wearer's back muscles. 


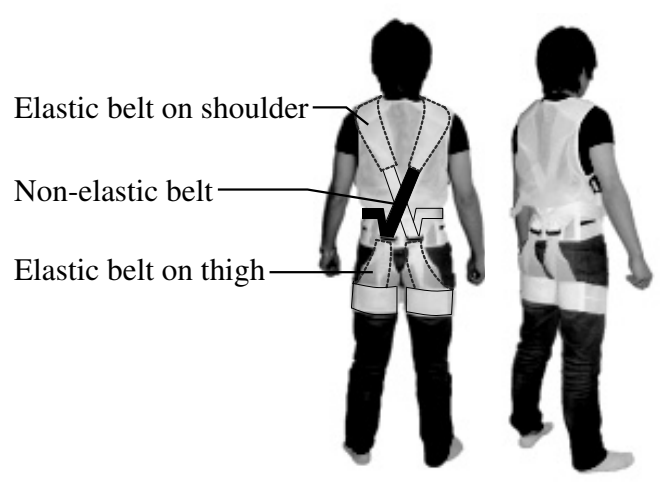

Fig. 3. Passive power-assist device "Smart Suite Lite".

In the development of SSL, a design method for the arrangement and the strength of its elastic belts was proposed based on a digital human model and motion analysis. The optimal placement of the elastic belts may vary depending on the target movement because different belt arrangements result in different directions and magnitudes of the generated force. The suit used in this paper was originally made for care workers ${ }^{19}$ and the details were modified to fit the physique of HRP-4. The measured values of the elastic properties of the belts on the shoulder were $232 \mathrm{~N} / \mathrm{m}$ and those of the belts on the thigh were $547 \mathrm{~N} / \mathrm{m}$.

\subsection{Generating an assistive torque estimation map}

This section describes the generation of the assistive torque map with regard to three degrees of freedom of the chest joint: the pitch angle $\theta_{p}$, the roll angle $\theta_{r}$, and the yaw angle $\theta_{y}$ (see Fig. 4). The structures of the chest and the lumbar region of the robot were simplified and different from those of the actual human body. This type of model is often used in biomechanical research to estimate the load on the lower back. ${ }^{25}$

Movements for measuring the torque were created discretely in the pitch, roll, and yaw of the chest joint within each range of motion. The discrete joint angles of the chest roll $\theta_{r}$ were $0^{\circ}, \pm 6.6^{\circ}, \pm 13.4^{\circ}$, and $\pm 20.0^{\circ}$, and those of the chest yaw $\theta_{y}$ were $0^{\circ}, \pm 10.0^{\circ}, \pm 20.0^{\circ}$, and $\pm 30.0^{\circ}$. For the combination of these joint angles, the chestpitch joint $\theta_{p}$ was slowly moved continuously from $-7.1^{\circ}$ to $24.8^{\circ}$. The other joint angles were as shown in Fig. 4.

We measured the joint torques once for each movement by using internal sensors under quasi-static conditions and under wearing/non-wearing conditions. The following nonlinear polynomial was assumed as a relational expression between torques and angles:

$$
\hat{\tau}_{l}\left(\theta_{r}, \theta_{p}, \theta_{y}\right)=\Sigma_{(0 \leq i+j+k \leq 3)} a_{i j k}\left(\theta_{r}\right)^{i}\left(\theta_{p}\right)^{j}\left(\theta_{y}\right)^{k},
$$




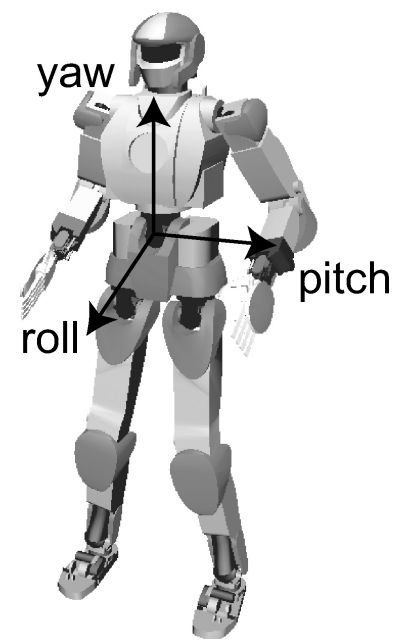

Fig. 4. Definition of a rotation axis of each joint.

where $\hat{\tau}_{l}$ is the estimated value of the joint torque $(l=r, p, y)$, and $i, j$, and $k$ denote the order of each variable and are the integers greater than or equal to zero. The coefficient $a_{i j k}$ were determined by the least squares method. The number of the unknown coefficients was 20, and the number of the measurement data was 34,349 points. Figure 5 is an example of the measured joint torque and the curved surface,

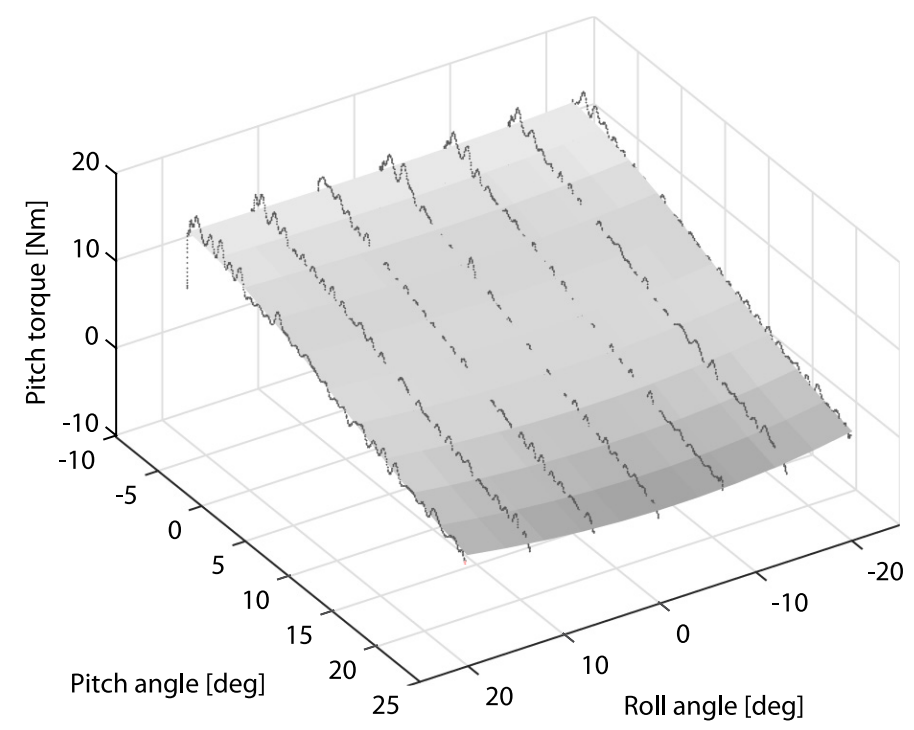

Fig. 5. An example of the nonlinear curve-fitting of the measured joint torque (chest-pitch torque, during flexion phase, chest yaw angle $=0^{\circ}$ ). The curved surface shows the fitting result, the point cloud represents the measured value. 
which is the fitting result of the pitch joint torque with respect to the angle of the pitch joint and the roll joint. In addition, the estimation equation for the assistive torque $\hat{\tau}_{\text {la }}$ is defined as follows:

$$
\hat{\tau}_{\text {la }}=\hat{\tau}_{\text {ln }}-\hat{\tau}_{\text {ls }},
$$

where $\hat{\tau}_{\text {ln }}$ is the estimation equation under normal condition, and $\hat{\tau}_{\text {ls }}$ is the estimation equation for the wearing SSL condition. This estimation equation is defined as the assistive torque estimation map on the chest joint. The root-mean-square error (RMSE) between the measured torques and the assistive torque estimation map was $1.22 \mathrm{Nm}$ in the pitch torque, $0.48 \mathrm{Nm}$ in the roll torque, and $0.90 \mathrm{Nm}$ in the yaw torque. The range of the measured assist torque was $10.35 \mathrm{Nm}$ in the pitch, $6.33 \mathrm{Nm}$ in the roll, and $21.81 \mathrm{Nm}$ in the yaw. The estimation map could estimate the assistive effects accurately within an error of approximately $12 \%$.

\subsection{Validation with retargeted human motion}

We can estimate the assistive effect for arbitrary motions by using the map constructed in Sec. 3.2. To validate this, the estimation and the measurement of the assistive torque were performed with motions that were created based on the human motion. The following two motions were recorded by the motion capture system and converted to the humanoid robot motion using the motion retargeting method. ${ }^{20}$ The retargeting method reproduces the whole-body motion as close to human motions as possible with a humanoid robot. The human motions are measured by using a motion capture system and converted to feasible motions for a humanoid robot. As there exists the difference of body structure between the robot and the human, the method deals with the identification problem of the morphing function between the human model and the robot model at same time.

(i) Twisting motion (Fig. 6). This involves lifting up the imaginary object that is placed at the right lower position and putting it on the left higher position by twisting the waist, and then putting it back to the original position.

(ii) Transfer motion (Fig. 7). This motion reproduces a nursing care work called "transferring". It involves bending forward and lifting up the care-receiver from the bed and then transferring the person to the chair placed by the side.
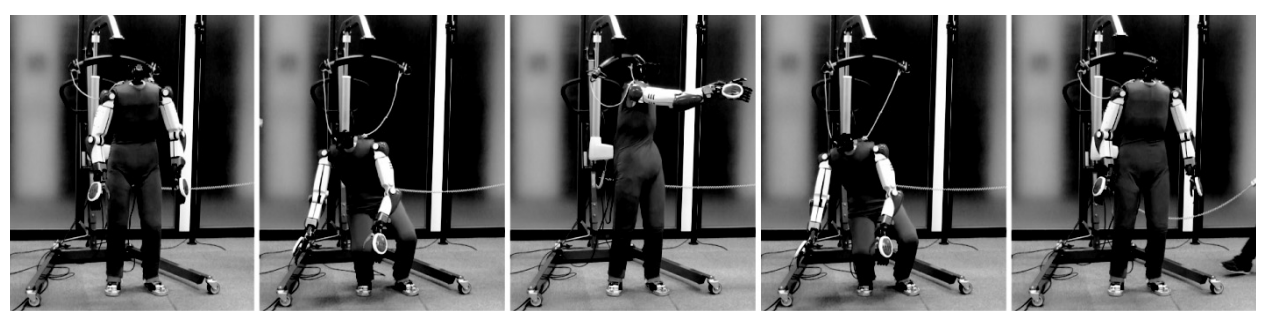

Fig. 6. Snapshots of the twisting motion of HRP-4. 

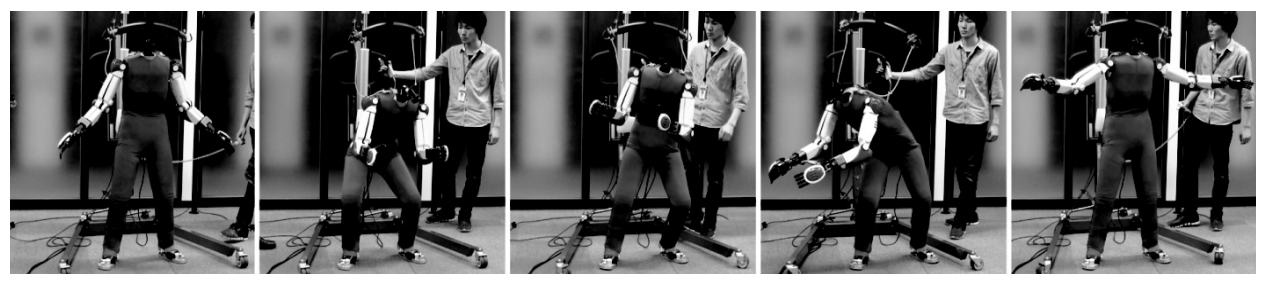

Fig. 7. Snapshots of the transfer motion of HRP-4.

The chest joint angles during the motion are shown in Figs. 8 and 9. Figures 10 and 11 show the measured value of the torque of each axis. The change in torque are not so large because SSL has been developed under the concept that the suit should not degrade the user's physical function by excessive assistance. Figures 12 and 13 show the assistive torques estimated by the torque estimation map and the measured

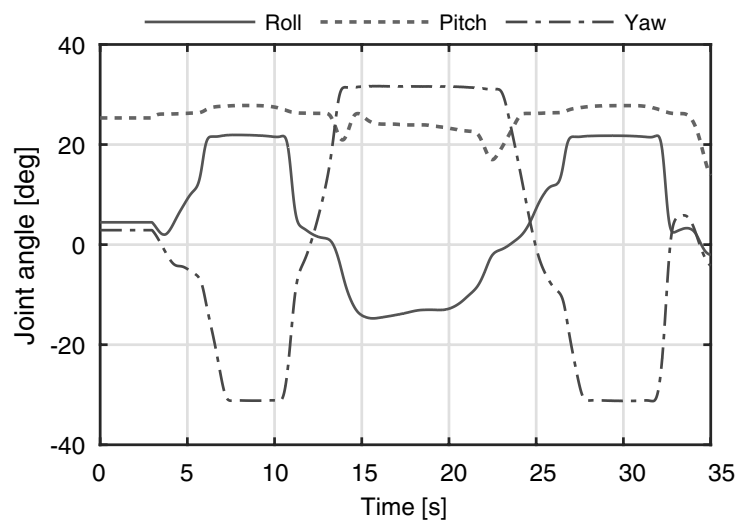

Fig. 8. Chest joint angle in twisting motion.

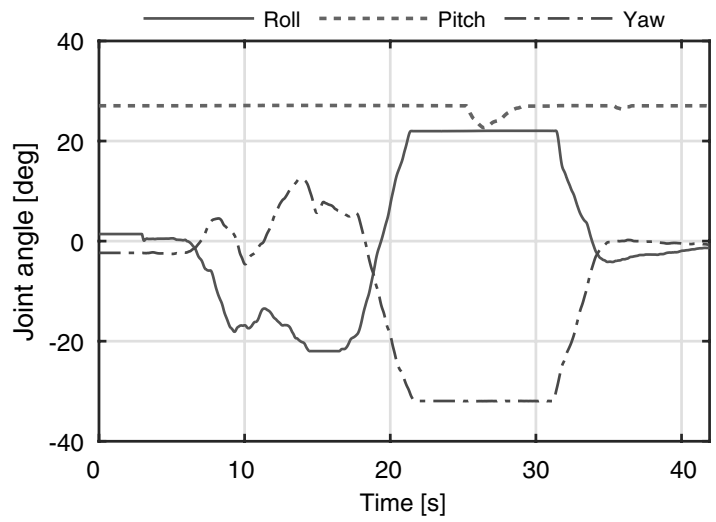

Fig. 9. Chest joint angle in transfer motion. 


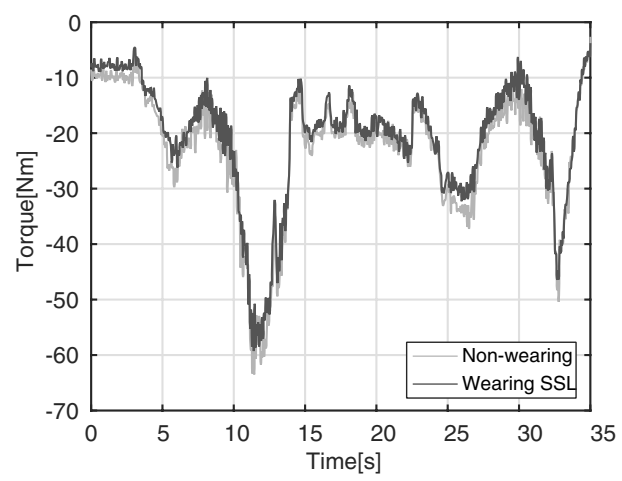

(a) Chest pitch

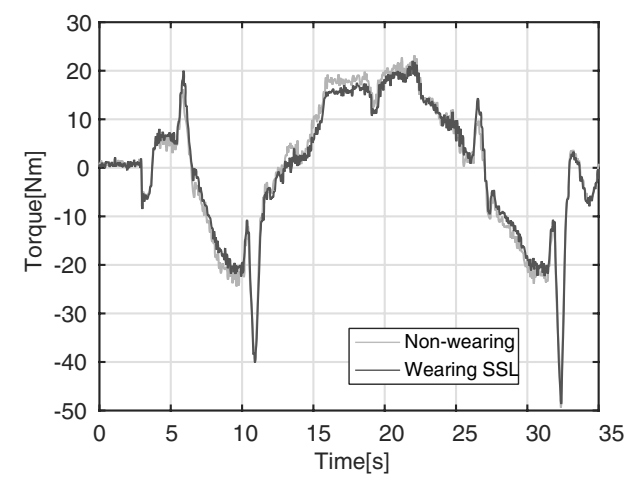

(b) Chest roll

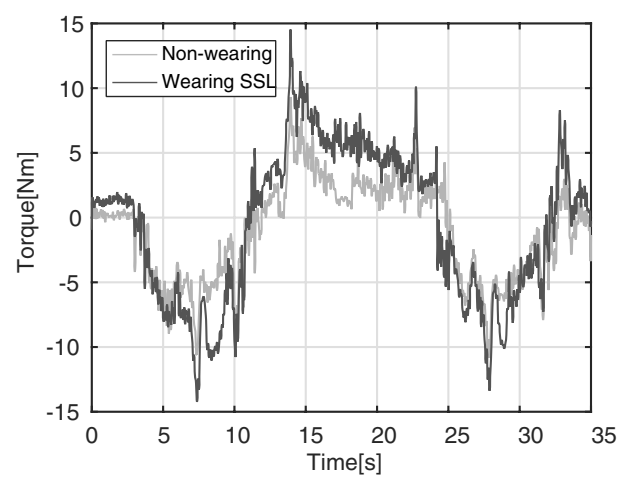

(c) Chest yaw

Fig. 10. Measured joint torques during the twisting motion.

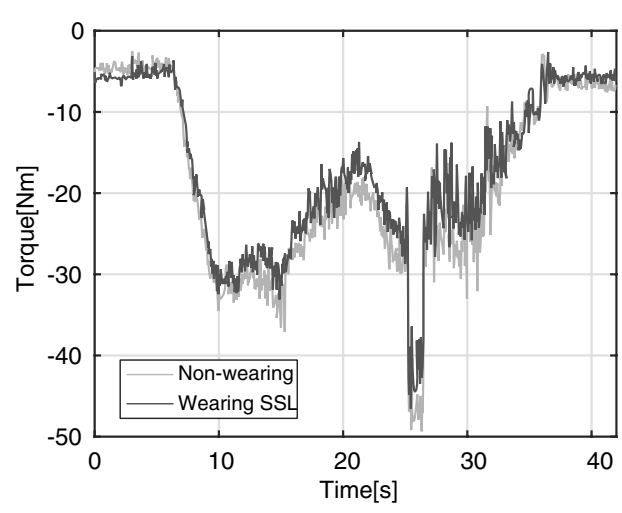

(a) Chest pitch

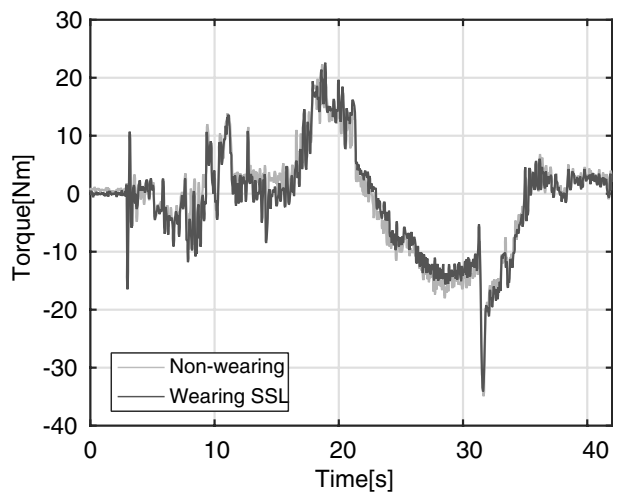

(b) Chest roll

Fig. 11. Measured joint torques during the transfer motion. 
Y. Imamura et al.

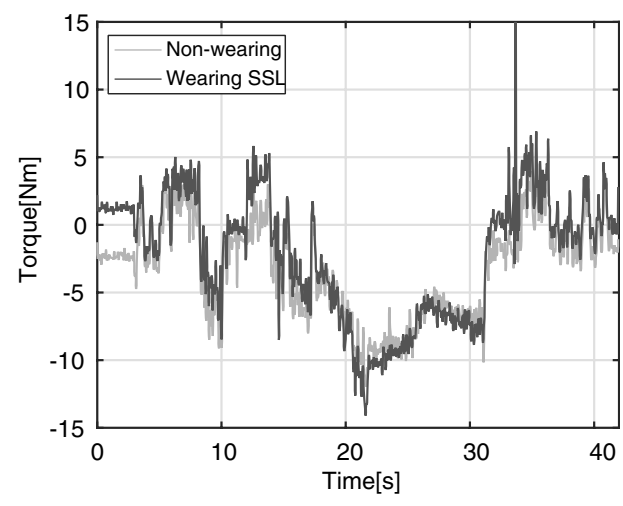

(c) Chest yaw

Fig. 11. (Continued)

assistive torques. In the twisting motion, the RMSE of the pitch torque was $2.28 \mathrm{Nm}$, the roll torque was $1.28 \mathrm{Nm}$, and the yaw torque was $2.33 \mathrm{Nm}$. Also, in the twisting motion, the RMSE of the pitch torque was $2.52 \mathrm{Nm}$, the roll torque was $1.60 \mathrm{Nm}$, and the yaw torque was $2.99 \mathrm{Nm}$.

From Figs. 12 and 13, it is seen that the accuracy of torque estimation in the chest pitch torque is lower at a section from $15 \mathrm{~s}$ to $22 \mathrm{~s}$ in the twisting motion, and at the first $7 \mathrm{~s}$ and the last $7 \mathrm{~s}$ in the transfer motion. We consider that this decrease in accuracy was caused by the movement of the hip joints. The angle of the hip pitch joint is shown in Figs. 14 and 15. Since SSL is structured to connect the back and the leg, it is influenced by the motion of the leg during whole-body movement. The pitch joint angles of the hip when generating the estimation map were about $-10^{\circ}$; which means slight flexion posture, in both legs. Considering this $-10^{\circ}$ as the reference posture, the hip joint angles were smaller than the reference posture in the

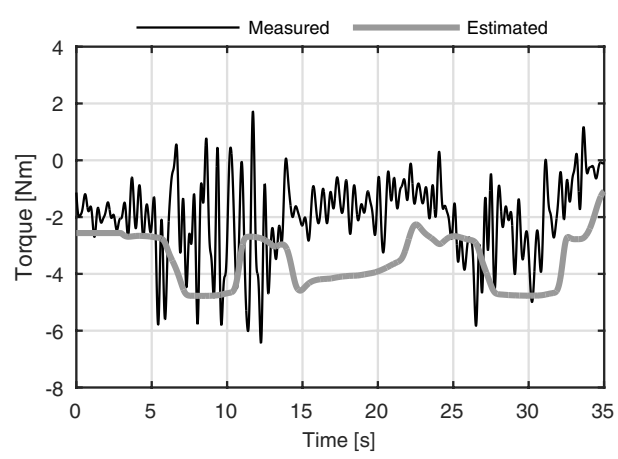

(a) Chest pitch

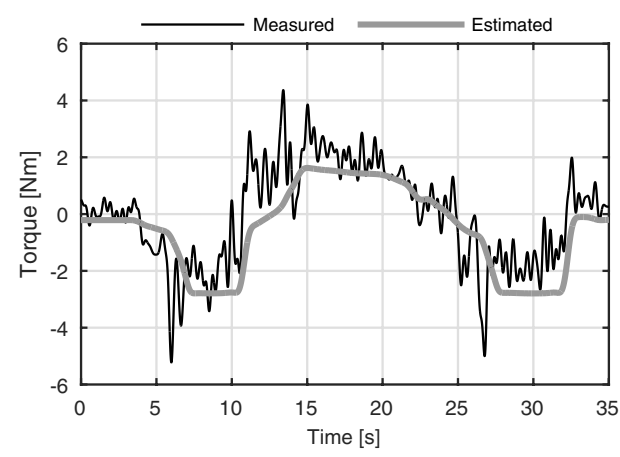

(b) Chest roll

Fig. 12. Comparison of the measured and estimated assistive torques during the twisting motion. 


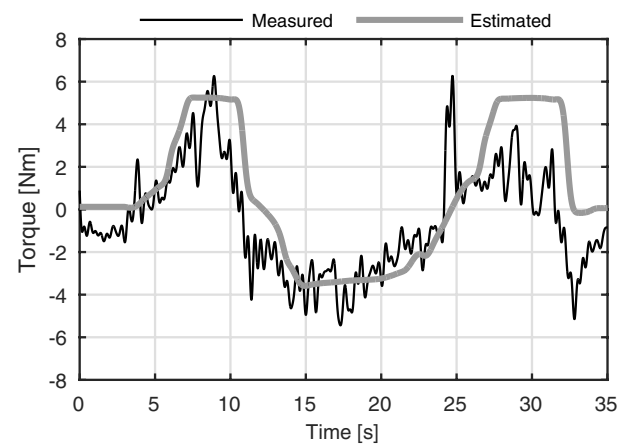

(c) Chest yaw

Fig. 12. (Continued)

above-mentioned low-accuracy sections, which means that both hip joints were extended. As a result, it can be considered that the elastic materials were slackened because of the movement of the hip joints and the assistive forces were decreased. When creating the torque estimation map, we did not take into account such a slack

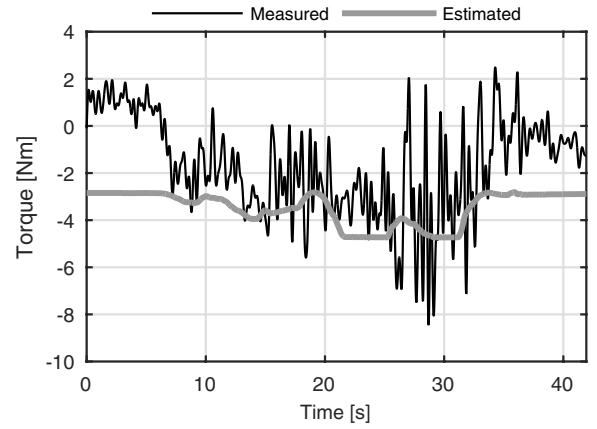

(a) Chest pitch

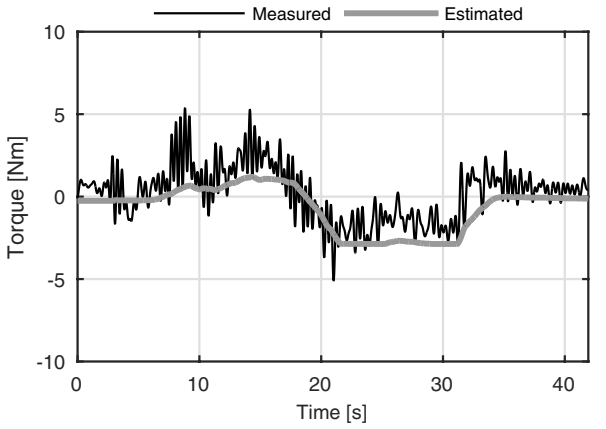

(b) Chest roll

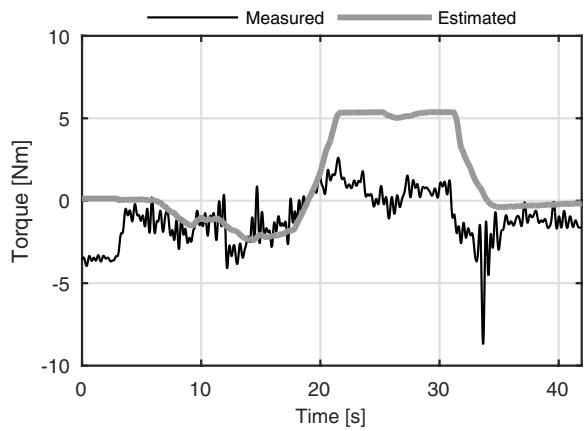

(c) Chest yaw

Fig. 13. Comparison of the measured and estimated assistive torques during the transfer motion. 


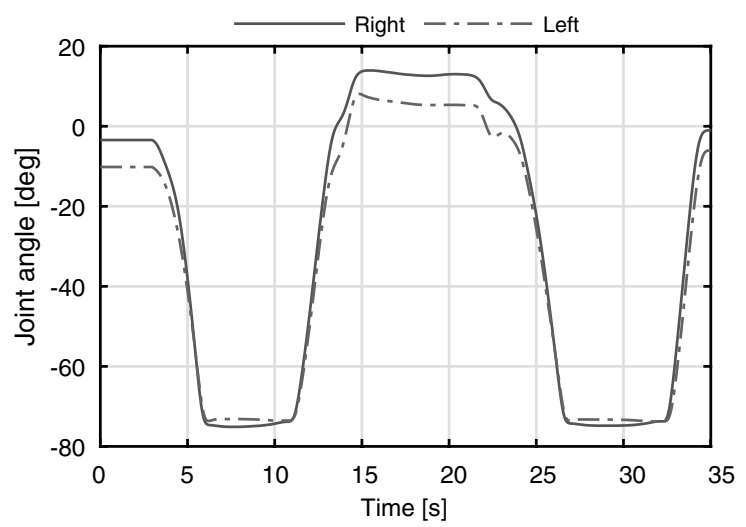

Fig. 14. Pitch angle of the hip joint in twisting motion.

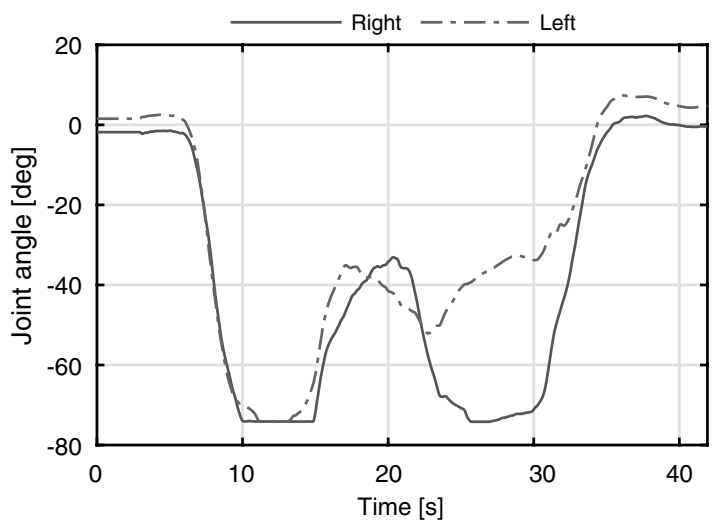

Fig. 15. Pitch angle of the hip joint in transfer motion.

of the suit, so we found that an error is caused for the posture when bending the upper body backward.

In addition, the assistive torque disappeared in the yaw joint of the transfer motion from $20 \mathrm{~s}$ to $30 \mathrm{~s}$. We consider that the path of the elastic material changed due to the significant twist of the body including the movement of the leg, and resulted in the change in the moment arm. Figure 16 shows the measurement result of the assistive torque when only the angle of the chest joint was actuated and the angle of the hip joints were fixed during the transfer motion. In this case, the assistive torque was closer to the expected value. The elastic belt applied from the left shoulder to the right leg generates a positive torque on the yaw joint. It is considered that the moment arm sufficient to generate the yaw torque was lost as a result of the path of this elastic belt shifting to the right due to the bending of the right hip. 


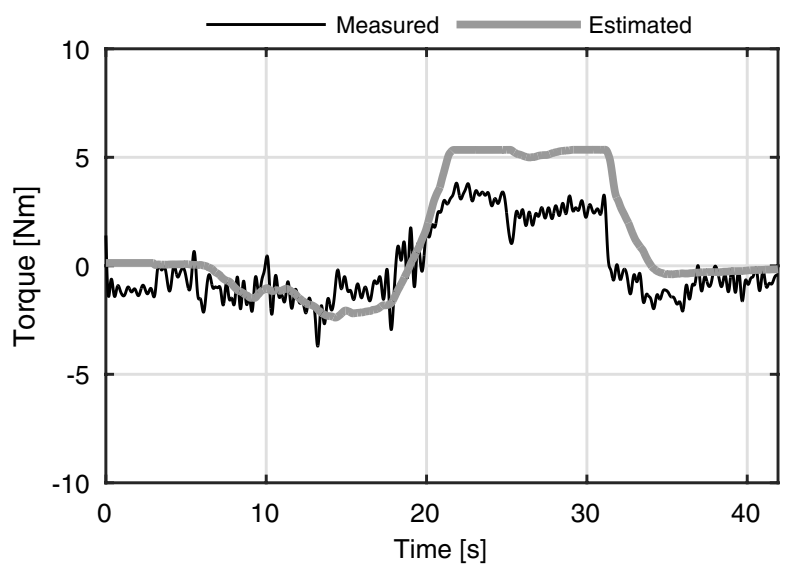

Fig. 16. Comparison of the measured and estimated assistive torques during the transfer motion. In this measurement, the chest joint was activated while the others including hip joints were kept fixed in the initial position.

Although the estimation map is generated by discrete and static measurements, it was confirmed that the map can reproduce the patterns of the assistive torque against the complex motion when the motion mainly consists of the chest joint movement. Since the estimation errors of the torque were found when the other joints also had a great influence on the motion, considering the comprehensive movement of the involved joint to construct the torque estimation map will be a problem to be resolved in the future.

\section{Evaluation of Geometric Simulation Model Using Torque Estimation Map}

In this section, we evaluate the geometric simulation models as one application example of the proposed torque estimation map. The assistive torque estimation map is useful for evaluating the actual device, on the other hand, a geometric simulation model is also required when estimating the effect of the design change or the change in the user's body shape. In this section, estimation maps were generated by using simulation results with geometric models as the dataset. Those generated maps were evaluated by comparing with the assistive torque estimation map based on the actual measurement. The assistive torque estimation map was utilized as the reference value in this section.

\subsection{Simulation model}

This section describes a model that represents the elastic materials as wires placed on the three-dimensional model of the robot for the assistive effect estimation. 


\section{Y. Imamura et al.}

The passive power-assist suit was modeled with wires in the same way as a musculoskeletal modeling: the joint torques are obtained from the muscles modeled as wires. $^{26}$ We can use the same methodology to simulate the elastic bands of the passive power-assist suit by modeling with the tension spring wires. ${ }^{19}$ The wires have several via-points fixed on the surface of the computer-aided design (CAD) model of the robot (see Fig. 17). The assistive torque could be calculated as the product of a change in the path length, moment arms, and elastic coefficient. The changes in the path lengths are obtained for the two wires, respectively, in the model. In addition, the moment arms of the two wires are calculated for the chest roll joint, the pitch joint, and the yaw joint, respectively. The elastic coefficient is determined from the elasticity coefficients of the elastic belt on the shoulder and the elastic belt on the thigh region, because these two elastic belts are combined in a unique structure in one wire path. Details are shown in Ref. 15. The elastic coefficient of each wire is $172 \mathrm{~N} / \mathrm{m}$ in this model.

In this paper, we prepared two types of the wire model, named "wire model A" and "wire model B". The wire model A is the original model used in the previous paper. ${ }^{15}$ Our previous research revealed that this model had low reproducibility against the twisting motion. Therefore, we made another wire model, named as wire model B, based on the three-dimensional-shape measurements using the optical motion capture system (MAC3D, Motion Analysis Corp.). The wire path was determined by using the measurement results of several optical markers placed on the robot and the elastic belts of SSL, as shown in Fig. 18. The wire placements of the model A and model B are shown in Fig. 19. The wire model B is expected to have a higher estimation accuracy because it is based on the actual shape measurement.

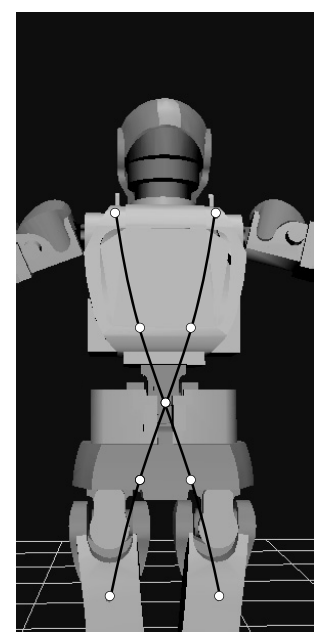

Fig. 17. Wire path model on the robot's CAD model. 
Small circle: Optical markers on the elastic belts

$\bigcirc$ Large circle: Optical markers on the feature points of the robot

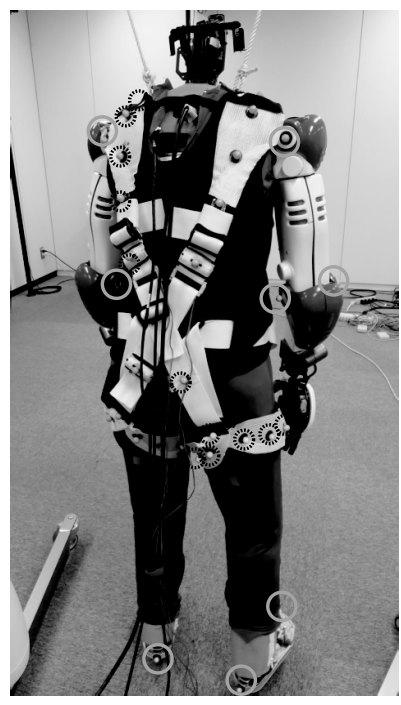

(a)

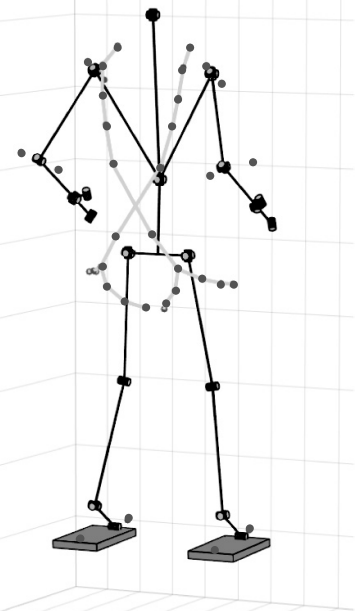

(b)

Fig. 18. Experiment measuring the three-dimensional shape of the elastic wire path: (a) Experiment scene. (b) Result of the measurement.

\subsection{Comparison with the torque estimation map}

We created additional torque estimation maps by using wire models for comparison. The discrete joint angles of the chest roll were $0^{\circ}, \pm 6.6^{\circ}, \pm 13.4^{\circ}$, and $\pm 20.0^{\circ}$, those of the chest yaw were $0^{\circ}, \pm 10.0^{\circ}, \pm 20.0^{\circ}$, and $\pm 30.0^{\circ}$, and those of the chest pitch

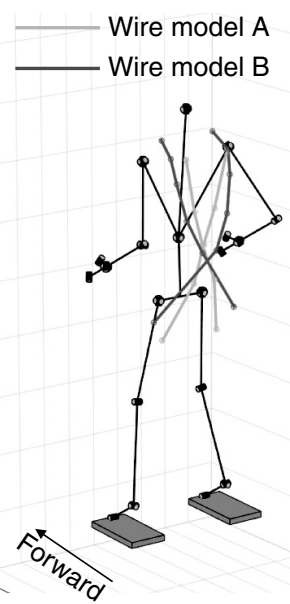

Fig. 19. Wire path models. 


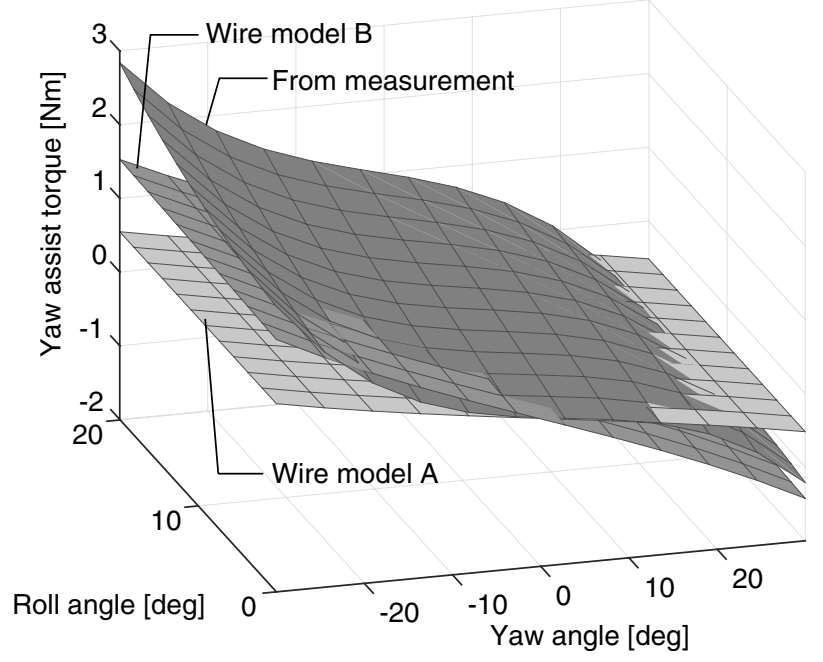

Fig. 20. Comparison in assistive torque estimation map: original torque estimation map based on the measurement, and estimation maps generated from the wire model A and the wire model B.

were $-7^{\circ},-1.7^{\circ}, 3.7^{\circ}, 9.0^{\circ}, 14.3^{\circ}, 19.7^{\circ}$, and $25.0^{\circ}$. The assistive torques were estimated for each posture by using two types of the wire models mentioned in Sec. 4.1, and these assistive torque estimation maps were created by using Eq. (1). Figure 20 shows the examples of the created maps, which represent the fitting result of the yaw joint torque with respect to the angles of the yaw joint and the roll joint. It can be seen that a large yaw angle causes a great error especially in the wire model A, which showed a low reproducibility against the twisting motion in the previous paper. As compared with the wire model A, the wire model B is closer to the torque estimation map created from the measured values.

Table 1 shows the RMSE of the two types of wire model estimations from the original torque estimation map. No difference is observed between the two types with respect to the pitch joint; however, the errors of the wire model B are reduced with respect to the roll and yaw joints. Here, the original torque estimation map is assumed to include a deviation from the measured torque, (The RMSE is $0.48 \mathrm{Nm}$ for the roll torque, and $0.90 \mathrm{Nm}$ for the yaw torque.) The RMSE of the wire model A exceeds the original torque error both in the roll joint and the yaw joint. However, the results show that the RSME of the wire model B is suppressed within the

Table 1. RMSE of the wire model and torque estimation map $[\mathrm{Nm}]$.

\begin{tabular}{lcc}
\hline & Wire model A & Wire model B \\
\hline Pitch & 0.77 & 0.8 \\
Roll & 0.65 & 0.45 \\
Yaw & 1.11 & 0.57 \\
\hline
\end{tabular}


variation of the original map model. For the pitch joint, the original estimation accuracy was not low, and it has been confirmed in the previous humanoid robot experiment. ${ }^{15}$ Therefore, no improvement was found by the modification of this time. This means that the wire model B may be superior with respect to the twisting motion, including the roll and the yaw.

\subsection{Model validation with retargeted human motion}

Finally, the accuracy of estimating the wire models is verified with the motion used in Sec. 3.3. In this experiment, the chest joints were activated while the others were kept fixed in the initial position to avoid changes in the support force by the movement of the other joints. Figures 21 and 22 show the comparison between the actual measurement value and the estimated assist torque by using the joint angles of each motion as an input to the wire models. In addition, Table 2 shows RMSE of the wire model estimation with respect to the measured value. The figures and the table show that the wire model $\mathrm{B}$ is able to reproduce the tendency of the change in the assistive torque with respect to the roll joint and the yaw joint. From these results,

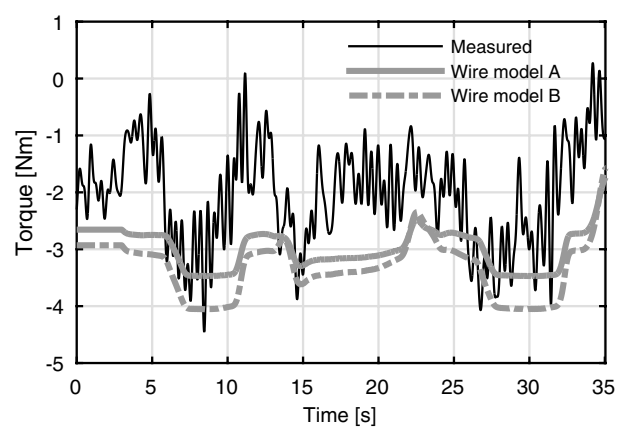

(a) Chest pitch

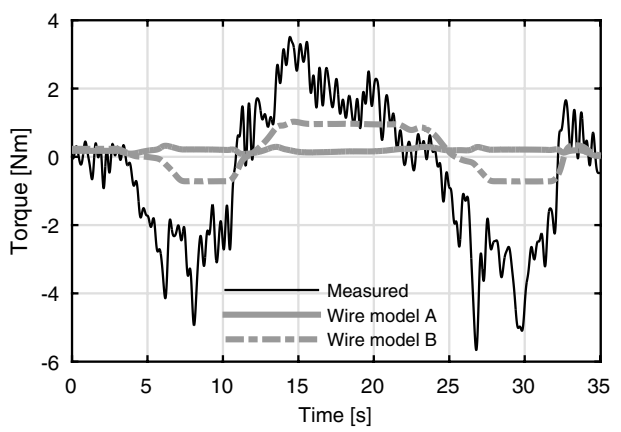

(b) Chest roll

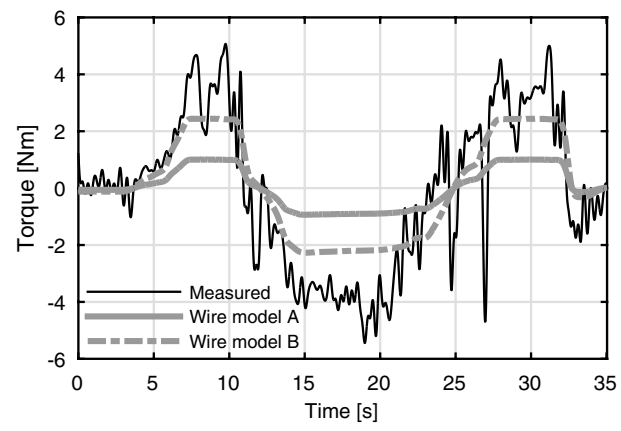

(c) Chest yaw

Fig. 21. Comparison of the measured and estimated assistive torques by the wire model during the twisting motion. 


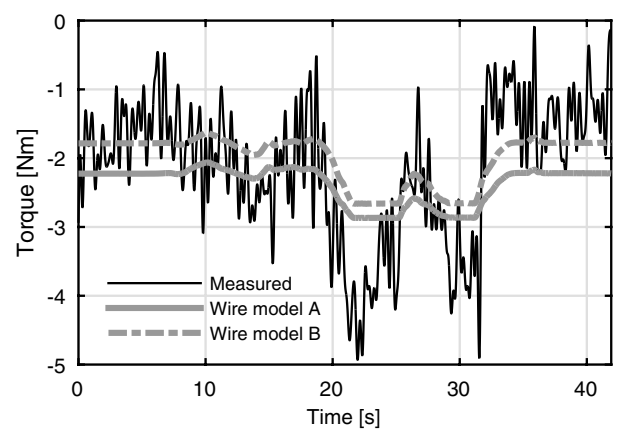

(a) Chest pitch

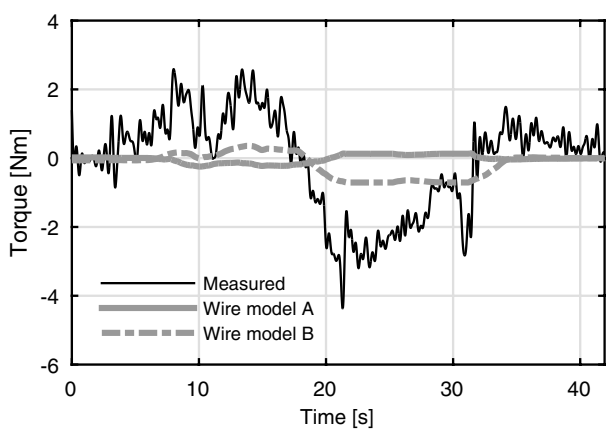

(b) Chest roll

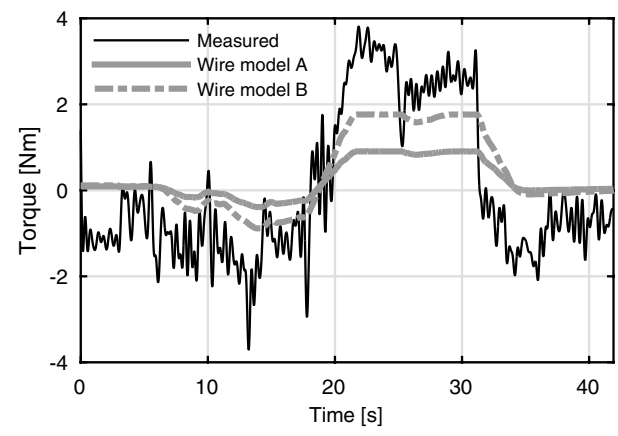

(c) Chest yaw

Fig. 22. Comparison of the measured and estimated assistive torques by the wire model during the transfer motion.

it was found that the wire model B increased the reproducibility of the assistive effects for complex twisting movements. This is consistent with the result of comparison between the assistive torque estimation maps mentioned in Sec. 4.2. We can conclude that the accuracy of the geometric simulation model can be evaluated by using a static torque estimation map without conducting experiments of complex motion, and we can select the more appropriate model.

Table 2. RMSE of the simulated assistive torque.

\begin{tabular}{llcc}
\hline & & Wire model A & Wire model B \\
\hline Twisting & Pitch & 1.29 & 1.54 \\
& Roll & 2.19 & 1.68 \\
& Yaw & 2.12 & 1.44 \\
Transfer & Pitch & 0.92 & 0.85 \\
& Roll & 1.56 & 1.20 \\
& Yaw & 1.45 & 1.17 \\
\hline
\end{tabular}




\section{Conclusions}

In this study, we presented a new method to estimate the assistive effect of the passive assistive device by using a humanoid robot, and we evaluated the simulation model by using the proposed method. This approach can construct the estimation model directly from the measurement data of the humanoid robot. The advantage of this method is that it does not depend on specific motions, and it does not require knowledge about the device. It is also possible to estimate the assistive effects for the other motion without performing the actual measurement experiments by using the devices.

In our previous work, although simulation and experiment torques were in good agreement, they were only valid for a particular motion. To apply the torque estimation to a wider range of motions, we employed a set of postures that covered the range of motion of the target joint. By performing actual measurements of the joint torque of the humanoid robot, we created the torque estimation map that represented the relationship between the joint angles and the assistive torque. The map can estimate the assistive torque during the movement within the range of motion. However, the model was generated by using the chest joint movement; therefore, it does not consider the influence of the other joints. In more complex movements, the other joints affect the assistive force. Therefore, in our future work, it is necessary to study the torque estimation map based on the comprehensive movement of the involved joints.

In addition, we described the evaluation of the geometric simulation model of SSL by using the estimation map of the assistive torque. The target motions were complex in the previous verification experiment; therefore, it was difficult to examine the quantitative error of each degree of freedom or to identify the cause of the modeling errors. In the proposed method, a comprehensive estimation map of the assistive torque is created in advance, and it covers the possible combinations of the postures of the target joint. The modeling error can be quantitatively evaluated within the range of joint motion by comparing the torque estimation map and the simulation model. In the experiment, we compared the simulation results with two geometric models and the measured assistive torque. As a result, the simulation model closer to the torque estimation map was also verified to have greater estimation accuracy for the complicated human-like motion. A comparison with the torque estimation map was useful to evaluate the simulation model of the assistive devices.

Limitation on evaluation with the humanoid robot is that it could not deal with the physiological and psychological evaluations and the individual differences in physiques. To address the individual differences in height, weight, age, sex, and degree of fitness, it is desirable to create an individual digital human model and perform simulation, because mass production of human-sized robots is difficult in practice. On the other hand, only the human-subject experiment can perform psychological and physiological measurements. Therefore, it is necessary to combine several evaluations appropriately for designing and evaluating the device in 
consideration of the merits and demerits of each of the simulation, the robot evaluation, and the subject experiment.

\section{Acknowledgment}

This research was partly supported by the METI Robotic Devices for the Nursing Care Project.

\section{References}

1. Y. Sankai, Hal: Hybrid assistive limb based on cybernics, Robotics Research 66 (2010) $25-34$.

2. H. Kobayashi, New robot technology concept applicable to human physical support The concept and possibility of the muscle suit, J. Robot. Mechatron. 14 (2002) 46-53.

3. H. Herr, Exoskeletons and orthoses: Classification, design challenges and future directions, J. Neuroeng. Rehabil. 6(21) (2009).

4. A. B. Zoss, H. Kazerooni and A. Chu, Biomechanical design of the Berkeley lower extremity exoskeleton (BLEEX), in IEEE/ASME Trans. Mechatronics 11(2) (2006) $128-138$.

5. K. Kiguchi, Active exoskeletons for upper-limb motion assist, Int. J. Hum. Robot. 4(3) (2007) 607-624.

6. T. Tanaka, Y. Satoh, S. Kaneko, Y. Suzuki and N. Sakamoto, S. Seki, Smart suit: Soft power suit with semi-active assist mechanism-prototype for supporting waist and knee joint, in Int. Conf. Control, Automation and Systems (ICCAS) (IEEE, Seoul, Korea, 2008), pp. 2002-2005.

7. ISO 13482:2014, Robots and robotic devices - Safety requirements for personal care robots, http://www.iso.org/iso/home/store/catalogue_tc/catalogue_detail.htm? csnumber $=53820$.

8. K. Anam and A. A. Al-Jumaily, Active exoskeleton control systems: State-of-the-art, Procedia Eng. 41 (2012) 988-994.

9. Y. Muramatsu, H. Umehara and H. Kobayashi, Improvement and quantitative performance estimation of the back support muscle suit, in 35th Annual Int. Conf. of the IEEE Engineering in Medicine and Biology Society (EMBC) (Osaka, Japan, 2013), pp. 28442849.

10. Y. Muramatsu and H. Kobayashi, Assessment of local muscle fatigue by NIRS-development and evaluation of muscle suit, Robomech J. 1(19) (2014).

11. Y. Kurita, J. Sato, T. Tanaka, M. Shinohara and T. Tsuji, Unloading muscle activation enhances force perception, in 5th Augmented Human Int. Conf. (ACM, Kobe, Japan, 2014).

12. Y. Imamura, T. Tanaka and Y. Suzuki, Analysis of trunk stabilization effect by passive power-assist device, J. Robot. Mechatron. 26(6) (2014), 791-798.

13. K. Miura, E. Yoshida, Y. Kobayashi, Y. Endo, F. Kanehioro, K. Homma, I. Kajitani, Y. Matsumoto and T. Tanaka, Humanoid robot as an evaluator of assistive devices, in Int. Conf. Robotics and Automation (ICRA) (IEEE, Karlsruhe, Germany, 2013), pp. 679-685.

14. K. Ayusawa, S. Nakaoka, E. Yoshida, Y. Imamura and T. Tanaka, Evaluation of assistive devices using humanoid robot with mechanical parameters identification, in IEEE-RAS 14 th Int. Conf. Humanoid Robots (Humanoids) (IEEE, Madrid, Spain, 2014), pp. 205211. 
15. Y. Imamura, T. Tanaka, K. Ayusawa and E. Yoshida, Verification of passive power-assist device using humanoid robot: Effect on bending and twisting motion, in IEEE-RAS 15th Int. Conf. Humanoid Robots (Humanoids) (IEEE, Seoul, Korea, 2015), pp. 1149-1154.

16. K. Ayusawa, E. Yoshida, Y. Imamura and T. Tanaka, New evaluation framework for human-assistive devices based on humanoid robotics, Adv. Robot. 30(8) (2016) $519-534$.

17. H. Kondo, Y. Ogura, H. Aikawa, A. Morishima, J. Shimizu, H.-O. Lim and A. Takanishi, Application of biped humanoid robot to motion simulation for elderly and disabled people, in 6th Int. Conf. International Society for Gerontechnology (ISG, Tuscany, Italy, 2008).

18. G. Nelson, A. Saunders, N. Neville, B. Swilling, J. Bondaryk, D. Billings, C. Lee, R. Playter and M. Raibert, Petman: A humanoid robot for testing chemical protective clothing, J. Robot. Soc. Jpn. 30(4) (2012) 372-377.

19. Y. Imamura, T. Tanaka, Y. Suzuki, K. Takizawa and M. Yamanaka, Motion-baseddesign of elastic material for passive assistive device using musculoskeletal model, $J$. Robot. Mechatron. 23(6) (2011) 978-990.

20. K. Ayusawa, M. Morisawa and E. Yoshida, Motion retargeting for humanoid robots based on identification to preserve and reproduce human motion features, in 2015 IEEE/ RSJ Int. Conf. Intelligent Robots and Systems (IROS) (IEEE, Hamburg, Germany, 2015), pp. 2774-2779.

21. K. Kaneko, F. Kanehiro, M. Morisawa, K. Akachi, G. Miyamori, A. Hayashi and N. Kanehira, Humanoid robot HRP-4-humanoid robotics platform with lightweight and slim body, in 2011 IEEE/RSJ Int. Conf. Intelligent Robots and Systems (IROS) (IEEE, San Francisco, USA, 2011), pp. 4400-4407.

22. K. Kaneko, F. Kanehiro, M. Morisawa, K. Miura, S. Nakaoka and S. Kajita, Cybernetic human HRP-4c, in 9th IEEE-RAS Int. Conf. Humanoid Robots (Humanoids) (IEEE, Paris, France, 2009), pp. 7-14.

23. S. R. Hamner, A. Seth and S. L. Delp, Muscle contributions to propulsion and support during running, J. Biomech. 43(14) (2010) 2709-2716.

24. N. I. Badler, C. B. Phillips and B. L. Webber, Simulating Humans: Computer Graphics Animation and Control (Oxford University Press, Oxford, 1999).

25. P. L. Santaguida, M. Pierrynowski, C. Goldsmith and G. Fernie, Comparison of cumulative low backloads of caregivers when transferring patients using overhead and floor mechanical lifting devices, Clin. Biomech. 20(9) (2005) 906-916.

26. Y. Nakamura, K. Yamane, Y. Fujita and I. Suzuki, Somatosensory computation for manmachine interface from motion-capture data and musculoskeletal human model, IEEE Trans. Robot. 21(1) (2005) 58-66. 


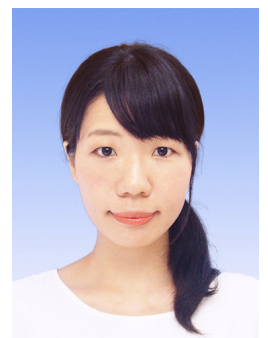

Yumeko Imamura received the Master degree of Information Science and Technology and Ph.D. in the field of Systems Science and Informatics from the Graduate School of Information Science and Technology, Hokkaido University, in 2011 and 2014, respectively. From 2014-2015, she served as a Research Fellow of Japan Society for the Promotion of Science at Hokkaido University. She is currently a Researcher of CNRS-AIST Joint Robotics Laboratory, UMI3218/RL, and Intelligent Systems Research Institute (IS-AIST), National Institute of Advanced Industrial Science and Technology (AIST), Japan.

Her research interests include biomechanics, human modeling, and power assistive technologies.

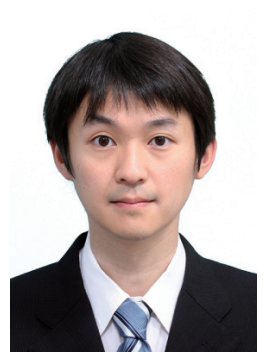

Ko Ayusawa received the B.Sc. degree in Mechanical Engineering, and the M.Sc. and Ph.D. degrees in Mechano-Informatics from the University of Tokyo, Japan, in 2006, 2008 and 2011, respectively. He worked with the Department of MechanoInformatics at the University of Tokyo, as a Postdoctoral Researcher from 2011-2012, and as a Project Assistant Processor in 2013. He is currently a Researcher of Intelligent Systems Research Institute, National Institute of Advanced Industrial Science and Technology (AIST), Tsukuba, Japan, and a Researcher of CNRS-AIST JRL (Joint Robotics Laboratory), UMI3218/RL.

His research interests include the identification of human/humanoid dynamics, motion control for humanoid robots, and kinematics and dynamics simulation for human musculoskeletal models.

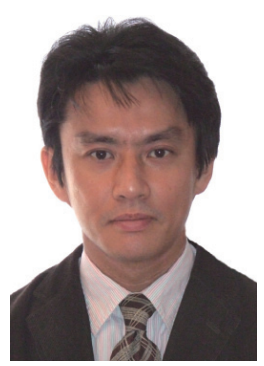

Eiichi Yoshida received the M.Engrg. and Ph.D. degrees on Precision Machinery Engineering from the Graduate School of Engineering, the University of Tokyo in 1993 and 1996, respectively. In 1996 he joined the former Mechanical Engineering Laboratory, later reorganized as the National Institute of Advanced Industrial Science and Technology (AIST), Tsukuba, Japan. He served as the Co-Director of AIST/IS-CNRS/ST2I Joint French-Japanese Robotics Laboratory (JRL) at LAASCNRS, Toulouse, France, from 2004-2008. Since 2009, he is the Co-Director of CNRS-AIST JRL (Joint Robotics Laboratory), UMI3218/RL, and since 2015 he serves as the Deputy-Director of Intelligent Systems Research Institute (IS-AIST), AIST, Tsukuba, Japan.

His research interests include robot task and motion planning, human modeling, and humanoid robots. 


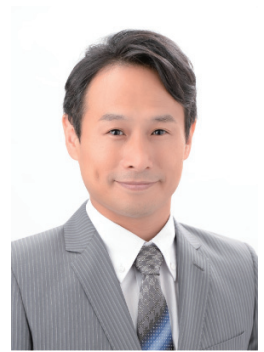

Takayuki Tanaka received the B.C. degree in 1994, the M.Sc. degree in 1996, and the Ph.D. degree, respectively, from the University of Electro-Communications (UEC). He was an Assistant Professor of the Department Mechanical and Control Engineering of UEC from April 1996-March 2003. During this period, He was also a Visiting Researcher of the University of California, Irvine through October 2001-March 2002. He became an Associate Professor of UEC in April 2003. Since April 2004, he is currently an Associate Professor of the Graduate School of Information Science and Technology of the Hokkaido University.

His major research topics are assistive robot, wearable robot, and human sensing. He is a member of RSJ, SICE, IEEE, and a fellow member of JSME. 